Korean J. Math. 20 (2012), No. 2, pp. 193-212

\title{
THE PROPERTIES OF FUZZY CONNECTIONS
}

\author{
Yong Chan KIm* and Young Sun Kim
}

\begin{abstract}
We investigate the properties of fuzzy connections. We find generating functions which induce fuzzy connections. In particular, we show that their connections relate to fuzzy relations.
\end{abstract}

\section{Introduction}

Wille [7] introduced the formal concept lattices by allowing some uncertainty in data as examples as Galois, dual Galois, residuated and dual residuated connections. Formal concept analysis is an important mathematical tool for data analysis and knowledge processing [1-4,7]. Orlowska and Rewitzky [5] investigated the algebraic structures of operators of Galois-style connections. Bělohlávek [1-2] introduced the formal concept lattices with respect to fuzzy Galois connections on a complete residuated lattice. Fuzzy Galois connections are developed many directions $[3,4,8]$

In this paper, we investigate the properties of fuzzy connections (Galois, dual Galois, residuated and dual residuated connections) on a complete residuated lattice. We find generating functions which induce fuzzy connections (Galois, dual Galois, residuated and dual residuated connections). In particular, we show that their connections relate to fuzzy relations.

\section{Preliminaries}

Definition 2.1. $[1,2,6]$ A triple $(X, \leq, \odot)$ is called a complete residuated lattice iff it satisfies the following properties:

\footnotetext{
Received April 11, 2012. Revised June 1, 2012. Accepted June 5, 2012.

2010 Mathematics Subject Classification: 06A06, 06A15, 06B30, 54F05,68U35.

Key words and phrases: complete residuated lattice, fuzzy connections (Galois, dual Galois, residuated and dual residuated connections).

* Corresponding author.
} 
(L1) $(X, \leq, 1,0)$ is a complete lattice where 1 is the universal upper bound and 0 denotes the universal lower bound;

(L2) $(X, \odot, 1)$ is a commutative monoid;

(L3) $\odot$ is distributive over arbitrary joins, i.e.

$$
\left(\bigvee_{i \in \Gamma} x_{i}\right) \odot y=\bigvee_{i \in \Gamma}\left(x_{i} \odot y\right), \forall x_{i}, y \in L
$$

Let $(L, \leq, \odot)$ be a complete residuated lattice. For each $x, y \in L$, we define

$$
x \rightarrow y=\bigvee\{z \in L \mid x \odot z \leq y\} .
$$

Then it satisfies Galois correspondence, that is,

$(x \odot y) \leq z$ iff $x \leq(y \rightarrow z)$.

REMARK 2.2. [1,2,6](1) A completely distributive lattice is a complete residuated lattice. Moreover, the unit interval $([0,1], \leq, \vee, \wedge, 0,1)$ is a complete residuated lattice.

(2) The unit interval with a left-continuous t-norm $t,([0,1], \leq, t)$, is a complete residuated lattice.

Let $(L, \leq, \odot)$ be a complete residuated lattice. An order reversing map $^{*}: L \rightarrow L$ defined by $a^{*}=a \rightarrow 0$ is called a strong negation if $a^{* *}=a$ for each $a \in L$.

In this paper, we assume $\left(L, \leq, \odot,^{*}\right)$ is a complete residuated lattice with a strong negation *. For $\alpha \in L, A, 1_{x} \in L^{X}$, we denote

$$
\begin{gathered}
(\alpha \odot A)(x)=\alpha \odot A(x), \quad(\alpha \rightarrow A)(x)=\alpha \rightarrow A(x) . \\
1_{x}(x)=1,1_{x}(y)=0, \forall y \in X-\{x\} .
\end{gathered}
$$

Lemma 2.3. [6] For each $x, y, z, x_{i}, y_{i} \in L$, we have the following properties.

(1) If $y \leq z, x \odot y \leq x \odot z, x \rightarrow y \leq x \rightarrow z$ and $z \rightarrow x \leq y \rightarrow x$.

(2) $x \odot y \leq x \wedge y$.

(3) $x \rightarrow\left(\bigwedge_{i \in \Gamma} y_{i}\right)=\bigwedge_{i \in \Gamma}\left(x \rightarrow y_{i}\right)$ and $\left(\bigvee_{i \in \Gamma} x_{i}\right) \rightarrow y=\bigwedge_{i \in \Gamma}\left(x_{i} \rightarrow\right.$ $y)$.

(4) $x \rightarrow\left(\bigvee_{i \in \Gamma} y_{i}\right) \geq \bigvee_{i \in \Gamma}\left(x \rightarrow y_{i}\right)$

(5) $\left(\bigwedge_{i \in \Gamma} x_{i}\right) \rightarrow y \geq \bigvee_{i \in \Gamma}\left(x_{i} \rightarrow y\right)$.

(6) $(x \odot y) \rightarrow z=x \rightarrow(y \rightarrow z)=y \rightarrow(x \rightarrow z)$. 
(7) $x \odot(x \rightarrow y) \leq y$ and $x \rightarrow y \leq(y \rightarrow z) \rightarrow(x \rightarrow z)$.

(8) $y \odot z \leq x \rightarrow(x \odot y \odot z)$ and $x \odot(x \odot y \rightarrow z) \leq y \rightarrow z$.

(9) $x \rightarrow y \leq(x \odot z) \rightarrow(y \odot z)$.

(10) $(x \rightarrow y) \odot(y \rightarrow z) \leq x \rightarrow z$.

(11) $x \leq y \rightarrow z$ iff $y \leq x \rightarrow z$.

(12) $x \rightarrow y=1$ iff $x \leq y$.

(13) $x \rightarrow y=y^{*} \rightarrow x^{*}$.

(14) $\bigwedge_{i \in \Gamma} x_{i}^{*}=\left(\bigvee_{i \in \Gamma} x_{i}\right)^{*}$ and $\bigvee_{i \in \Gamma} x_{i}^{*}=\left(\bigwedge_{i \in \Gamma} x_{i}\right)^{*}$

Definition 2.4. [8] Let $X$ be a set. A function $e_{X}: X \times X \rightarrow L$ is called a fuzzy partially order on $X$ if it satisfies the following conditions:

(E1) $e_{X}(x, x)=1$ for all $x \in X$,

(E2) $e_{X}(x, y) \odot e_{X}(y, z) \leq e_{X}(x, z)$, for all $x, y, z \in X$,

(E3) if $e_{X}(x, y)=e_{X}(y, x)=1$, then $x=y$.

The pair $\left(X, e_{X}\right)$ is a fuzzy partially order set (simply, fuzzy poset).

We define a function $e_{L^{X}}: L^{X} \times L^{X} \rightarrow L$ as

$$
e_{L^{X}}(A, B)=\bigwedge_{x \in X}(A(x) \rightarrow B(x)) .
$$

Then $\left(L^{X}, e_{L^{x}}\right)$ is a fuzzy poset from Lemma $2.3(10-11)$.

We define fuzzy connections in a sense [8].

Definition 2.5. Let $\left(X, e_{X}\right)$ and $\left(Y, e_{Y}\right)$ be fuzzy posets and $f$ : $X \rightarrow Y$ and $g: Y \rightarrow X$ maps.

(1) $\left(e_{X}, f, g, e_{Y}\right)$ is called a Galois connection if

$$
e_{Y}(y, f(x))=e_{X}(x, g(y)), \forall x \in X, y \in Y .
$$

(2) $\left(e_{X}, f, g, e_{Y}\right)$ is called a dual Galois connection if

$$
e_{Y}(f(x), y)=e_{X}(g(y), x), \forall x \in X, y \in Y .
$$

(3) $\left(e_{X}, f, g, e_{Y}\right)$ is called a residuated connection if

$$
e_{Y}(f(x), y)=e_{X}(x, g(y)), \forall x \in X, y \in Y .
$$

(4) $\left(e_{X}, f, g, e_{Y}\right)$ is called a dual residuated connection if

$$
e_{Y}(y, f(x))=e_{X}(g(y), x), \forall x \in X, y \in Y .
$$




\section{The properties of fuzzy connections}

TheOrem 3.1. The following statements hold:

(1) There exists a Galois connection $\left(e_{L^{X}}, F, G, e_{L^{Y}}\right)$ iff there exists a function $F: L^{X} \rightarrow L^{Y}$ with $F\left(\bigvee_{i \in \Gamma} A_{i}\right)=\bigwedge_{i \in \Gamma} F\left(A_{i}\right)$ and $F(\alpha \odot A)=$ $\alpha \rightarrow F(A)$ and $F\left(1_{x}\right)(y)=G\left(1_{y}\right)(x), \forall x \in X, y \in Y$.

(2) There exists a residuated connection $\left(e_{L^{X}}, F, G, e_{L^{Y}}\right)$ iff there exists a function $F: L^{X} \rightarrow L^{Y}$ with $F\left(\bigvee_{i \in \Gamma} A_{i}\right)=\bigvee_{i \in \Gamma} F\left(A_{i}\right)$ and $F(\alpha \odot A)=\alpha \odot F(A)$ and $F\left(1_{x}\right)^{*}(y)=G\left(1_{y}^{*}\right)(x), \forall x \in X, y \in Y$.

(3) There exists a dual Galois connection $\left(e_{L^{X}}, F, G, e_{L^{Y}}\right)$ iff there exists a function $F: L^{X} \rightarrow L^{Y}$ with $F\left(\bigwedge_{i \in \Gamma} A_{i}\right)=\bigvee_{i \in \Gamma} F\left(A_{i}\right), F(\alpha \rightarrow$ $A)=\alpha \odot F(A)$ and $F\left(1_{x}^{*}\right)(y)=G\left(1_{y}^{*}\right)(x), \forall x \in X, y \in Y$.

(4) There exists a dual residuated connection $\left(e_{L^{X}}, F, G, e_{L^{Y}}\right)$ iff there exists a function $F: L^{X} \rightarrow L^{Y}$ with $F\left(\bigwedge_{i \in \Gamma} A_{i}\right)=\bigwedge_{i \in \Gamma} F\left(A_{i}\right)$ and $F(\alpha \rightarrow A)=\alpha \rightarrow F(A)$ and $F\left(1_{x}^{*}\right)(y)=G\left(1_{y}\right)^{*}(x), \forall x \in X, y \in$ $Y$.

Proof. $(1)(\Rightarrow)$ By Lemma $2.3(3,6)$, we have

$$
\begin{aligned}
e_{L^{Y}}\left(B, F\left(\bigvee_{i \in \Gamma} A_{i}\right)\right) & =e_{L^{X}}\left(\bigvee_{i \in \Gamma} A_{i}, G(B)\right)=\bigwedge_{i \in \Gamma} e_{L^{X}}\left(A_{i}, G(B)\right) \\
& =\bigwedge_{i \in \Gamma} e_{L^{Y}}\left(B, F\left(A_{i}\right)\right)=e_{L^{Y}}\left(B, \bigwedge_{i \in \Gamma} F\left(A_{i}\right)\right), \\
e_{L^{Y}}(B, F(\alpha \odot A)) & =e_{L^{X}}(\alpha \odot A, G(B))=\alpha \rightarrow e_{L^{X}}(A, G(B)) \\
& =\alpha \rightarrow e_{L^{Y}}(B, F(A))=e_{L^{Y}}(\alpha \odot B, F(A)) \\
& =e_{L^{Y}}(B, \alpha \rightarrow F(A)) .
\end{aligned}
$$

For $B=1_{y} \in L^{Y}$

$$
\begin{aligned}
F\left(\bigvee_{i \in \Gamma} A_{i}\right)(y) & =e_{L^{Y}}\left(1_{y}, F\left(\bigvee_{i \in \Gamma} A_{i}\right)\right)=e_{L^{Y}}\left(1_{y}, \bigwedge_{i \in \Gamma} F\left(A_{i}\right)\right) \\
& =\bigwedge_{i \in \Gamma} F\left(A_{i}\right)(y), \\
F(\alpha \odot A)(y) & =e_{L^{Y}}\left(1_{y}, F(\alpha \odot A)\right)=e_{L^{Y}}\left(1_{y}, \alpha \rightarrow F(A)\right) \\
& =\alpha \rightarrow F(A)(y) .
\end{aligned}
$$

Moreover, $G\left(1_{y}\right)(x)=e_{L^{X}}\left(1_{x}, G\left(1_{y}\right)\right)=e_{L^{Y}}\left(1_{y}, F\left(1_{x}\right)\right)=F\left(1_{x}\right)(y)$. 
$(\Leftarrow)$ Since $C=\bigvee_{x \in X}\left(C(x) \odot 1_{x}\right)$, we have

$$
F(C)(y)=F\left(\bigvee_{x \in X}\left(C(x) \odot 1_{x}\right)\right)(y)=\bigwedge\left(C(x) \rightarrow F\left(1_{x}\right)(y)\right)
$$

We define a function $G: L^{Y} \rightarrow L^{X}$ with

$$
\begin{aligned}
& G(B)(x)=\bigvee\{C(x) \mid F(C) \geq B\} \\
& =\bigvee\left\{C(x) \mid \bigwedge\left(C(x) \rightarrow F\left(1_{x}\right)(y) \geq B(y)\right\}\right. \\
& \text { (by Lemma } 2.3(11) \text { ) } \\
& =\bigvee\left\{C(x) \mid \bigwedge\left(B(y) \rightarrow F\left(1_{x}\right)(y) \geq C(x)\right\}\right. \\
& =\bigwedge\left(B(y) \rightarrow F\left(1_{x}\right)(y)\right) \text {. } \\
& e_{L^{Y}}(B, F(A))=\bigwedge_{y \in Y}\left(B(y) \rightarrow F\left(\bigvee_{x \in X}\left(A(x) \odot 1_{x}\right)\right)(y)\right) \\
& =\bigwedge_{y \in Y}\left(B(y) \rightarrow \bigwedge_{x \in X}\left(A(x) \rightarrow F\left(1_{x}\right)(y)\right)\right) \\
& =\bigwedge_{y \in Y} \bigwedge_{x \in X}\left(B(y) \rightarrow\left(A(x) \rightarrow F\left(1_{x}\right)(y)\right)\right) \\
& \text { (by Lemma } 2.3(6) \text { ) } \\
& =\bigwedge_{x \in X}\left(A(x) \rightarrow \bigwedge_{y \in Y}\left(B(y) \rightarrow F\left(1_{x}\right)(y)\right)\right) \\
& =\bigwedge_{x \in X}(A(x) \rightarrow G(B)(x))=e_{L^{X}}(A, G(B)) . \\
& e_{L^{Y}}\left(F\left(\bigvee_{i \in \Gamma} A_{i}\right), B\right)=e_{L^{X}}\left(\bigvee_{i \in \Gamma} A_{i}, G(B)\right)=\bigwedge_{i \in \Gamma} e_{L^{X}}\left(A_{i}, G(B)\right), \\
& =\bigwedge_{i \in \Gamma} e_{L^{Y}}\left(F\left(A_{i}\right), B\right)=e_{L^{Y}}\left(\bigvee_{i \in \Gamma} F\left(A_{i}\right), B\right) \\
& e_{L^{Y}}(F(\alpha \odot A), B)=e_{L^{X}}(\alpha \odot A, G(B))=\alpha \rightarrow e_{L^{X}}(A, G(B)) \\
& =\alpha \rightarrow e_{L^{Y}}(F(A), B)=e_{L^{Y}}(\alpha \odot F(A), B) .
\end{aligned}
$$


For $B=1_{y}^{*} \in L^{Y}$, by Lemma $2.3(13), F\left(\bigvee_{i \in \Gamma} A_{i}\right)=\bigvee_{i \in \Gamma} F\left(A_{i}\right)$ from:

$$
\begin{aligned}
& e_{L^{Y}}\left(F\left(\bigvee_{i \in \Gamma} A_{i}\right), 1_{y}^{*}\right)=e_{L^{Y}}\left(1_{y}, F\left(\bigvee_{i \in \Gamma} A_{i}\right)^{*}\right)=F\left(\bigvee_{i \in \Gamma} A_{i}\right)^{*}(y) \\
& e_{L^{Y}}\left(\bigvee_{i \in \Gamma} F\left(A_{i}\right), 1_{y}^{*}\right)=\left(\bigvee_{i \in \Gamma} F\left(A_{i}\right)\right)^{*}(y) .
\end{aligned}
$$

Similarly, $F(\alpha \odot A)=\alpha \odot F(A)$ for all $\alpha \in L$. Since $G\left(1_{y}^{*}\right) \in L^{X}$ and $F\left(1_{x}\right) \in L^{Y}, F\left(1_{x}\right)^{*}(y)=e_{L^{Y}}\left(1_{y}, F\left(1_{x}\right)^{*}\right)=e_{L^{Y}}\left(F\left(1_{x}\right), 1_{y}^{*}\right)=$ $e_{L^{X}}\left(1_{x}, G\left(1_{y}^{*}\right)\right)=G\left(1_{y}^{*}\right)(x)$.

$(\Leftarrow)$ Since $C=\bigvee_{x \in X}\left(C(x) \odot 1_{x}\right)$, we have

$$
F(C)(y)=F\left(\bigvee_{x \in X}\left(C(x) \odot 1_{x}\right)\right)(y)=\bigvee_{x \in X}\left(C(x) \odot F\left(1_{x}\right)(y)\right)
$$

We define a function $G: L^{Y} \rightarrow L^{X}$ with

$$
\begin{aligned}
G(B)(x)= & \bigvee\{C(x) \mid F(C) \leq B\} \\
= & \bigvee\left\{C(x) \mid \bigvee\left(C(x) \odot F\left(1_{x}\right)(y) \leq B(y)\right\}\right. \\
= & \bigwedge_{y \in Y}\left(F\left(1_{x}\right)(y) \rightarrow B(y)\right) . \\
e_{L^{Y}}(F(A), B) & =\bigwedge_{y \in Y}\left(F\left(\bigvee_{x \in X}\left(A(x) \odot 1_{x}\right)\right)(y) \rightarrow B(y)\right) \\
& =\bigwedge_{y \in Y}\left(\bigvee_{x \in X}\left(A(x) \odot F\left(1_{x}\right)(y) \rightarrow B(y)\right)\right. \\
& =\bigwedge_{y \in Y} \bigwedge_{x \in X}\left(A(x) \rightarrow\left(F\left(1_{x}\right)(y) \rightarrow B(y)\right)\right) \\
& =\bigwedge_{x \in X}\left(A(x) \rightarrow \bigwedge_{y \in Y}\left(F\left(1_{x}\right)(y) \rightarrow B(y)\right)\right) \\
& =\bigwedge_{x \in X}(A(x) \rightarrow G(B)(x))=e_{L^{X}}(A, G(B))
\end{aligned}
$$


$(3)(\Rightarrow)$

$$
\begin{aligned}
e_{L^{Y}}\left(F\left(\bigwedge_{i \in \Gamma} A_{i}\right), B\right) & =e_{L^{X}}\left(G(B), \bigwedge_{i \in \Gamma} A_{i}\right)=\bigwedge_{i \in \Gamma} e_{L^{X}}\left(G(B), A_{i}\right) \\
& =\bigwedge_{i \in \Gamma} e_{L^{Y}}\left(F\left(A_{i}\right), B\right)=e_{L^{Y}}\left(\bigvee_{i \in \Gamma} F\left(A_{i}\right), B\right) \\
e_{L^{Y}}(F(\alpha \rightarrow A), B) & =e_{L^{X}}(G(B), \alpha \rightarrow A)=\alpha \rightarrow e_{L^{X}}(G(B), A), \\
& =\alpha \rightarrow e_{L^{X}}(F(A), B)=e_{L^{X}}(\alpha \odot F(A), B) .
\end{aligned}
$$

For $B=1_{y}^{*} \in L^{Y}$, by a similar method as in (2), we have $F\left(\bigwedge_{i \in \Gamma} A_{i}\right)=$ $\bigvee_{i \in \Gamma} F\left(A_{i}\right)$ and $F(\alpha \rightarrow A)=\alpha \odot F(A)$.

$$
\begin{aligned}
F\left(1_{x}^{*}\right)^{*}(y) & =\bigwedge_{w \in Y}\left(1_{y}(w) \rightarrow F\left(1_{x}^{*}\right)^{*}(w)\right) \\
& =e_{L^{Y}}\left(F\left(1_{x}^{*}\right), 1_{y}^{*}\right)=e_{L^{X}}\left(G\left(1_{y}^{*}\right), 1_{x}^{*}\right) \\
& =G\left(1_{y}^{*}\right)^{*}(x) .
\end{aligned}
$$

$(\Leftarrow)$ For $A(x)=\bigwedge_{z \in X}\left(1_{z}(x) \rightarrow A(z)\right)=\bigwedge_{z \in X}\left(A^{*}(z) \rightarrow 1_{z}^{*}(x)\right)$, we have $F(A)(y)=F\left(\bigwedge_{z \in X}\left(A^{*}(z) \rightarrow 1_{z}^{*}\right)\right)(y)=\bigvee_{z \in X}\left(A^{*}(z) \odot F\left(1_{z}^{*}\right)(y)\right)$, we define

$$
\begin{aligned}
G(B)(x) & =\bigwedge\{C(x) \mid F(C) \leq B\} \\
& =\bigwedge\left\{C(x) \mid F\left(\bigwedge_{x \in X}\left(C^{*}(x) \rightarrow 1_{x}^{*}\right)\right) \leq B\right\} \\
& =\bigvee\left\{C(x) \mid \bigvee_{x \in X}\left(C^{*}(x) \odot F\left(1_{x}^{*}\right)(y) \leq B(y)\right\}\right. \\
& =\bigvee\left\{C(x) \mid C^{*}(x) \leq \bigwedge_{y \in Y}\left(F\left(1_{x}^{*}\right)(y) \rightarrow B(y)\right)\right\} \\
& =\bigvee_{y \in Y}\left(F\left(1_{x}^{*}\right)(y) \odot B^{*}(y)\right) .
\end{aligned}
$$




$$
\begin{aligned}
e_{L^{Y}}(F(A), B) & =\bigwedge_{y \in Y}\left(\bigvee_{x \in X}\left(A^{*}(x) \odot F\left(1_{x}^{*}\right)(y) \rightarrow B(y)\right)\right. \\
& =\bigwedge_{y \in Y} \bigwedge_{x \in X}\left(\left(B^{*}(y) \odot F\left(1_{x}^{*}\right)(y) \rightarrow A(x)\right)\right. \\
& =\bigwedge_{x \in X}\left(\bigvee_{y \in Y}\left(B^{*}(y) \odot F\left(1_{x}^{*}\right)(y) \rightarrow A(x)\right)\right. \\
& =\bigwedge_{x \in X}(G(B)(x) \rightarrow A(x))=e_{L^{X}}(G(B), A)
\end{aligned}
$$

$(4)(\Rightarrow)$ We have:

$$
\begin{aligned}
& e_{L^{Y}}\left(B, F\left(\bigwedge_{i \in \Gamma} A_{i}\right)\right)=e_{L^{X}}\left(G(B), \bigwedge_{i \in \Gamma} A_{i}\right)=\bigwedge_{i \in \Gamma} e_{L^{X}}\left(G(B), A_{i}\right) \\
& =\bigwedge_{i \in \Gamma} e_{L^{Y}}\left(B, F\left(A_{i}\right)\right)=e_{L^{Y}}\left(B, \bigwedge_{i \in \Gamma} F\left(A_{i}\right)\right) \\
& e_{L^{Y}}(B, F(\alpha \rightarrow A))=e_{L^{X}}(G(B), \alpha \rightarrow A)=e_{L^{X}}(\alpha \odot G(B), A) \\
& =\alpha \rightarrow e_{L^{X}}(G(B), A)=\alpha \rightarrow e_{L^{X}}(B, F(A))=e_{L^{X}}(B, \alpha \rightarrow F(A))
\end{aligned}
$$

For $B=1_{y} \in L^{Y}$, by a similar method as in (1), we have $F\left(\bigwedge_{i \in \Gamma} A_{i}\right)=$ $\bigwedge_{i \in \Gamma} F\left(A_{i}\right)$ and $F(\alpha \rightarrow A)=\alpha \rightarrow F(A)$.

$$
\begin{aligned}
F\left(1_{x}^{*}\right)(y) & =\bigwedge_{w \in Y}\left(1_{y}(w) \rightarrow F\left(1_{x}^{*}\right)(w)\right) \\
& =e_{L^{Y}}\left(1_{y}, F\left(1_{x}^{*}\right)\right)=e_{L^{X}}\left(G\left(1_{y}\right), 1_{x}^{*}\right) \\
& =G\left(1_{y}\right)^{*}(x) .
\end{aligned}
$$

$(\Leftarrow)$ For $A(x)=\bigwedge_{z \in X}\left(1_{z}(x) \rightarrow A(z)\right)=\bigwedge_{z \in X}\left(A^{*}(z) \rightarrow 1_{z}^{*}(x)\right)$, we have $F(A)(y)=F\left(\bigwedge_{z \in X}\left(A^{*}(z) \rightarrow 1_{z}^{*}\right)\right)(y)=\bigwedge_{z \in X}\left(A^{*}(z) \rightarrow\right.$ 
$\left.F\left(1_{z}^{*}\right)(y)\right)$, we define

$$
\begin{aligned}
G(B)(x) & =\bigwedge\{C(x) \mid F(C) \geq B\} \\
& =\bigwedge\left\{C(x) \mid F\left(\bigwedge_{x \in X}\left(C^{*}(x) \rightarrow 1_{x}^{*}\right)\right) \geq B\right\} \\
& =\bigwedge\left\{C(x) \mid \bigwedge_{x \in X}\left(C^{*}(x) \rightarrow F\left(1_{x}^{*}\right)(y) \geq B(y)\right\}\right. \\
& =\bigwedge\left\{C(x) \mid C^{*}(x) \leq \bigwedge_{y \in Y}\left(B(y) \rightarrow F\left(1_{x}^{*}\right)(y)\right)\right\} \\
& =\bigwedge_{y \in Y}\left(F\left(1_{x}^{*}\right)^{*}(y) \odot B(y)\right) \\
e_{L^{Y}}(B, F(A)) & =\bigwedge_{y \in Y}\left(B(y) \rightarrow \bigwedge_{x \in X}\left(F\left(1_{x}^{*}\right)^{*}(y) \rightarrow A(x)\right)\right) \\
& =\bigwedge_{y \in Y} \bigwedge_{x \in X}\left(\left(B(y) \odot F\left(1_{x}^{*}\right)^{*}(y) \rightarrow A(x)\right)\right. \\
& =\bigwedge_{x \in X}\left(\bigvee_{y \in Y}\left(B(y) \odot F\left(1_{x}^{*}\right)^{*}(y) \rightarrow A(x)\right)\right. \\
& =\bigwedge_{x \in X}(G(B)(x) \rightarrow A(x))=e_{L^{X}}(G(B), A)
\end{aligned}
$$

THEOREM 3.2. (1) $\left(e_{L^{X}}, F, G, e_{L^{Y}}\right)$ is a Galois connection iff there exists $R: X \times Y \rightarrow L$ such that

$$
F(A)(y)=\bigwedge_{x \in X}(A(x) \rightarrow R(x, y)), \quad G(B)(x)=\bigwedge_{y \in Y}(B(y) \rightarrow R(x, y)) .
$$

(2) $\left(e_{L^{X}}, F, G, e_{L^{Y}}\right)$ is a residuated connection iff there exists $R$ : $X \times Y \rightarrow L$ such that

$$
F(A)(y)=\bigvee_{x \in X}(A(x) \odot R(x, y)), \quad G(B)(x)=\bigwedge_{y \in Y}(R(x, y) \rightarrow B(y))
$$


(3) $\left(e_{L^{x}}, F, G, e_{L^{Y}}\right)$ is a dual Galois connection iff there exists $R$ : $X \times Y \rightarrow L$ such that

$$
F(A)(y)=\bigvee_{x \in X}\left(A^{*}(x) \odot R(x, y)\right), \quad G(B)(x)=\bigwedge_{y \in Y}\left(R(x, y) \odot B^{*}(y)\right)
$$

(4) $\left(e_{L^{X}}, F, G, e_{L^{Y}}\right)$ is a dual residuated connection iff there exists $R \subset X \times Y$ such that

$$
F(A)(y)=\bigwedge_{y \in Y}(R(x, y) \rightarrow A(x)), \quad G(B)(x)=\bigvee_{y \in Y}(B(y) \odot R(x, y))
$$

Proof. $(1)(\Rightarrow)$

$$
\begin{aligned}
F\left(1_{x}\right)(y) & =e_{L^{Y}}\left(1_{y}, F\left(1_{x}\right)\right)=e_{L^{X}}\left(1_{x}, G\left(1_{y}\right)\right) \\
& =\bigwedge_{z \in X}\left(1_{x}(z) \rightarrow G\left(1_{y}\right)(z)\right)=G\left(1_{y}\right)(x) .
\end{aligned}
$$

Put $R(x, y)=F\left(1_{x}\right)(y)=G\left(1_{y}\right)(x)$. Then

$$
\begin{aligned}
\begin{aligned}
F(A)(y) & =e_{L^{Y}}\left(1_{y}, F(A)\right)=e_{L^{X}}\left(A, G\left(1_{y}\right)\right) \\
& =\bigwedge_{x \in X}\left(A(x) \rightarrow G\left(1_{y}\right)(x)\right) \\
& =\bigwedge_{x \in X}(A(x) \rightarrow R(x, y)) . \\
G(B)(x) & =e_{L^{X}}\left(1_{x}, G(B)\right)=e_{L^{Y}}\left(B, F\left(1_{x}\right)\right) \\
& =\bigwedge_{y \in Y}\left(B(y) \rightarrow F\left(1_{x}\right)(y)\right) \\
& =\bigwedge_{y \in Y}(B(y) \rightarrow R(x, y)) .
\end{aligned}
\end{aligned}
$$


$(\Leftarrow)$

$$
\begin{aligned}
e_{L^{Y}}(B, F(A)) & =\bigwedge_{y \in Y}(B(y) \rightarrow F(A)(y)) \\
& =\bigwedge_{y \in Y}\left(B(y) \rightarrow \bigwedge_{x \in X}(A(x) \rightarrow R(x, y))\right) \\
& =\bigwedge_{y \in Y} \bigwedge_{x \in X}(B(y) \rightarrow(A(x) \rightarrow R(x, y))) \\
& =\bigwedge_{x \in X}\left(A(x) \rightarrow \bigwedge_{y \in Y}(B(y) \rightarrow R(x, y))\right) \\
& =\bigwedge_{x \in X}(A(x) \rightarrow G(B)(x))=e_{L^{X}}(A, G(B)) .
\end{aligned}
$$

$(2)(\Rightarrow)$

$$
\begin{aligned}
F\left(1_{x}\right)^{*}(y) & =e_{L^{Y}}\left(F\left(1_{x}\right), 1_{y}^{*}\right)=e_{L^{X}}\left(1_{x}, G\left(1_{y}^{*}\right)\right) \\
& =G\left(1_{y}^{*}\right)(x) .
\end{aligned}
$$

Put $R(x, y)=F\left(1_{x}\right)(y)=\left(G\left(1_{y}^{*}\right)\right)^{*}$, then

$$
\begin{aligned}
F(A)^{*}(y) & =e_{L^{Y}}\left(F(A), 1_{y}^{*}\right)=e_{L^{X}}\left(A, G\left(1_{y}^{*}\right)\right) \\
& =\bigwedge_{x \in X}\left(A(x) \rightarrow G\left(1_{y}^{*}\right)(x)\right) \\
& =\left(\bigvee_{x \in X}\left(A(x) \odot G\left(1_{y}^{*}\right)^{*}(x)\right)\right)^{*} \\
F(A)(y) & =\bigvee_{x \in X}(A(x) \odot R(x, y)) . \\
G(B)(x) & =e_{L^{X}}\left(1_{x}, G(B)\right)=e_{L^{Y}}\left(F\left(1_{x}\right), B\right) \\
& =\bigwedge_{y \in Y}\left(F\left(1_{x}\right)(y) \rightarrow B(y)\right) \\
& =\bigwedge_{y \in Y}(R(x, y) \rightarrow B(y)) .
\end{aligned}
$$


$(\Leftarrow)$

$$
\begin{aligned}
e_{L^{Y}}(F(A), B) & =\bigwedge_{y \in Y}\left(\bigvee_{x \in X}(A(x) \odot R(x, y)) \rightarrow B(y)\right) \\
& =\bigwedge_{y \in Y} \bigwedge_{x \in X}(A(x) \rightarrow(R(x, y) \rightarrow B(y))) \\
& =\bigwedge_{x \in X}\left(A(x) \rightarrow \bigwedge_{y \in Y}(R(x, y) \rightarrow B(y))\right) \\
& =\bigwedge_{x \in X}(A(x) \rightarrow G(B)(x))=e_{L^{X}}(A, G(B)) .
\end{aligned}
$$

$(3)(\Rightarrow)$

$$
\begin{aligned}
F\left(1_{x}^{*}\right)^{*}(y) & =e_{L^{Y}}\left(F\left(1_{x}^{*}\right), 1_{y}^{*}\right)=e_{L^{X}}\left(G\left(1_{y}^{*}\right), 1_{x}^{*}\right) \\
& =G\left(1_{y}^{*}\right)^{*}(x) .
\end{aligned}
$$

Put $R(x, y)=F\left(1_{x}^{*}\right)(y)=G\left(1_{y}^{*}\right)(x)$, then

$$
\begin{aligned}
F(A)^{*}(y) & =e_{L^{Y}}\left(F(A), 1_{y}^{*}\right)=e_{L^{X}}\left(G\left(1_{y}^{*}\right), A\right) \\
& =\bigwedge_{x \in X}\left(G\left(1_{y}^{*}\right)(x) \rightarrow A(x)\right) \\
& =\left(\bigvee_{x \in X}\left(A^{*}(x) \odot G\left(1_{y}^{*}\right)(x)\right)\right)^{*} \\
F(A)(y) & =\bigvee_{x \in X}\left(A^{*}(x) \odot R(x, y)\right) . \\
G(B)^{*}(x) & =e_{L^{X}}\left(G(B), 1_{x}^{*}\right)=e_{L^{Y}}\left(F\left(1_{x}^{*}\right), B\right) \\
& =\bigwedge_{y \in Y}\left(F\left(1_{x}^{*}\right)(y) \rightarrow B(y)\right) \\
& =\left(\bigvee_{y \in Y}\left(B^{*}(y) \odot F\left(1_{x}^{*}\right)(y)\right)\right)^{*} \\
G(B)(x) & =\bigvee_{y \in Y}\left(B^{*}(y) \odot R(x, y)\right) .
\end{aligned}
$$


$(\Leftarrow)$

$$
\begin{aligned}
e_{L^{Y}}(F(A), B) & =\bigwedge_{y \in Y}\left(\bigvee_{x \in X}\left(A^{*}(x) \odot R(x, y)\right) \rightarrow B(y)\right) \\
& =\bigwedge_{y \in Y} \bigwedge_{x \in X}\left(\left(B^{*}(y) \odot R(x, y)\right) \rightarrow A(x)\right) \\
& =\bigwedge_{x \in X}\left(\bigvee_{y \in Y}\left(B^{*}(y) \odot R(x, y)\right) \rightarrow A(x)\right) \\
& =\bigwedge_{x \in X}(G(B)(x) \rightarrow A(x))=e_{L^{X}}(G(B), A)
\end{aligned}
$$

$(4)(\Rightarrow)$

$$
\begin{aligned}
F\left(1_{x}^{*}\right)(y) & =\bigwedge_{w \in Y}\left(1_{y}(w) \rightarrow F\left(1_{x}^{*}\right)(w)\right) \\
& =e_{L^{Y}}\left(1_{y}, F\left(1_{x}^{*}\right)\right)=e_{L^{X}}\left(G\left(1_{y}\right), 1_{x}^{*}\right) \\
& =G\left(1_{y}\right)^{*}(x) .
\end{aligned}
$$

Put $R(x, y)=F\left(1_{x}^{*}\right)^{*}(y)=G\left(1_{y}\right)$. We have

$$
\begin{aligned}
F(A)(y) & =e_{L^{Y}}\left(1_{y}, F(A)\right)=e_{L^{X}}\left(G\left(1_{y}\right), A\right) \\
& =\bigwedge_{x \in X}\left(G\left(1_{x}\right)(y) \rightarrow A(x)\right) \\
& =\bigwedge_{x \in X}(R(x, y) \rightarrow A(x)) . \\
G(B)^{*}(x) & =e_{L^{X}}\left(G(B), 1_{x}^{*}\right)=e_{L^{Y}}\left(B, F\left(1_{x}^{*}\right)\right) \\
& =\bigwedge_{y \in Y}\left(B(y) \rightarrow F\left(1_{x}^{*}\right)(y)\right) \\
& \left.=\bigvee_{y \in Y}\left(B(y) \odot F\left(1_{x}^{*}\right)^{*}(y)\right)\right)^{*} \\
G(B)(x) & =\bigvee_{y \in Y}(R(x, y) \odot B(y)) .
\end{aligned}
$$


$(\Leftarrow)$

$$
\begin{aligned}
e_{L^{Y}}(B, F(A)) & =\bigwedge_{y \in Y}\left(B(y) \rightarrow \bigwedge_{x \in X}(R(x, y) \rightarrow A(x))\right) \\
& =\bigwedge_{y \in Y} \bigwedge_{x \in X}((B(y) \odot R(x, y)) \rightarrow A(x)) \\
& =\bigwedge_{x \in X}\left(\bigvee_{y \in Y}(B(y) \odot R(x, y)) \rightarrow A(x)\right) \\
& =\bigwedge_{x \in X}(G(B)(x) \rightarrow A(x))=e_{L^{X}}(G(B), A) .
\end{aligned}
$$

THEOREM 3.3. (1) $\left(e_{L^{X}}, F, G, e_{L^{Y}}\right)$ is a Galois connection iff there exists $F: L^{X} \rightarrow L^{Y}$ with $F\left(1_{x}\right)(y)=\Gamma_{x}(y)$ such that $F\left(\bigvee_{i \in \Gamma} A_{i}\right)=$ $\bigwedge_{i \in \Gamma} F\left(A_{i}\right)$ and $F(\alpha \odot A)=\alpha \rightarrow F(A)$.

(2) $\left(e_{L^{X}}, F, G, e_{L^{Y}}\right)$ is a residuated connection iff there exists $F$ : $L^{X} \rightarrow L^{Y}$ with $F\left(1_{x}\right)(y)=\Gamma_{x}(y)$ such that $F\left(\bigvee_{i \in \Gamma} A_{i}\right)=\bigvee_{i \in \Gamma} F\left(A_{i}\right)$ and $F(\alpha \odot A)=\alpha \odot F(A)$.

(3) $\left(e_{L^{X}}, F, G, e_{L^{Y}}\right)$ is a dual Galois connection iff there exists $F$ : $L^{X} \rightarrow L^{Y}$ with $F\left(1_{x}^{*}\right)(y)=\Delta_{x}(y)$ such that $F\left(\bigwedge_{i \in \Gamma} A_{i}\right)=\bigvee_{i \in \Gamma} F\left(A_{i}\right)$ and $F(\alpha \rightarrow A)=\alpha \odot F(A)$.

(4) $\left(e_{L^{X}}, F, G, e_{L^{Y}}\right)$ is a dual residuated connection iff there exists $F: L^{X} \rightarrow L^{Y}$ with $F\left(1_{x}^{*}\right)(y)=\Delta_{x}(y)$ such that $F\left(\bigwedge_{i \in \Gamma} A_{i}\right)=$ $\bigwedge_{i \in \Gamma} F\left(A_{i}\right)$ and $F(\alpha \rightarrow A)=\alpha \rightarrow F(A)$.

Proof. (1) $(\Rightarrow)$ It follows from Theorem 3.1(1).

$(\Leftarrow)$ Since $C=\bigvee_{x \in X}\left(C(x) \odot 1_{x}\right), F(C)(y)=F\left(\bigvee_{x \in X}(C(x) \odot\right.$ $\left.\left.1_{x}\right)\right)(y)=\bigwedge\left(C(x) \rightarrow F\left(1_{x}\right)(y)\right)$. Thus,

$$
\begin{aligned}
G(B)(x) & =\bigvee\{C(x) \mid F(C) \geq B\} \\
& =\bigvee\left\{C(x) \mid \bigwedge\left(C(x) \rightarrow F\left(1_{x}\right)(y)\right) \geq B(y)\right\} \\
& =\bigvee\left\{C(x) \mid \bigwedge\left(B(y) \rightarrow F\left(1_{x}\right)(y)\right) \geq C(x)\right\} \\
& =\bigwedge\left(B(y) \rightarrow F\left(1_{x}\right)(y)\right)=\bigwedge\left(B(y) \rightarrow \Gamma_{x}(y)\right),
\end{aligned}
$$




$$
\begin{aligned}
e_{L^{Y}}(B, F(A)) & =\bigwedge_{y \in Y}\left(B(y) \rightarrow F\left(\bigvee_{x \in X}\left(A(x) \odot 1_{x}\right)\right)(y)\right) \\
& =\bigwedge_{y \in Y}\left(B(y) \rightarrow \bigwedge_{x \in X}\left(A(x) \rightarrow F\left(1_{x}\right)(y)\right)\right) \\
& =\bigwedge_{y \in Y} \bigwedge_{x \in X}\left(B(y) \rightarrow\left(A(x) \rightarrow F\left(1_{x}\right)(y)\right)\right) \\
& =\bigwedge_{x \in X}\left(A(x) \rightarrow \bigwedge_{y \in Y}\left(B(y) \rightarrow \Gamma_{x}(y)\right)\right) \\
& =\bigwedge_{x \in X}(A(x) \rightarrow G(B)(x))=e_{L^{Y}}(A, G(B))
\end{aligned}
$$

$(2)(\Rightarrow)$ It follows from Theorem 3.1(2).

$(\Leftarrow)$

$$
\begin{aligned}
G(B)(x)= & \bigvee\{C(x) \mid F(C) \leq B\} \\
= & \left\{C(x) \mid \bigvee\left(C(x) \odot F\left(1_{x}\right)\right)(y) \leq B(y)\right\} \\
= & \left.\bigwedge_{y \in Y}\left(F\left(1_{x}\right)\right)(y) \rightarrow B(y)\right)=\bigwedge_{y \in Y}\left(\Gamma_{x}(y) \rightarrow B(y)\right) . \\
e_{L^{Y}}(F(A), B) & \left.=\bigwedge_{y \in Y}\left(F\left(\bigvee_{x \in X}\left(A(x) \odot 1_{x}\right)\right)\right)(y) \rightarrow B(y)\right) \\
& =\bigwedge_{y \in Y}\left(\bigvee_{x \in X}\left(A(x) \odot F\left(1_{x}\right)\right)(y) \rightarrow B(y)\right) \\
& \left.=\bigwedge_{y \in Y} \bigwedge_{x \in X}\left(A(x) \rightarrow\left(F\left(1_{x}\right)\right)(y) \rightarrow B(y)\right)\right) \\
& =\bigwedge_{x \in X}\left(A(x) \rightarrow \bigwedge_{y \in Y}\left(\Gamma_{x}(y) \rightarrow B(y)\right)\right) \\
& =\bigwedge_{x \in X}(A(x) \rightarrow G(B)(x))=e_{L^{X}}(A, G(B)) .
\end{aligned}
$$

$(3)(\Rightarrow)$ It follows from Theorem 3.1(3). 


$$
\begin{aligned}
& (\Leftarrow) \text { Since } \\
& F\left(\bigwedge_{x \in X}\left(1_{x} \rightarrow C(x)\right)\right)=F\left(\bigwedge_{x \in X}\left(C^{*}(x) \rightarrow 1_{x}^{*}\right)=\bigvee\left(C^{*}(x) \odot F\left(1_{x}^{*}\right)\right),\right.
\end{aligned}
$$

we define

$$
\begin{aligned}
G(B)(x)= & \bigwedge\{C(x) \mid F(C) \leq B\} \\
= & \bigwedge\left\{C(x) \mid F\left(\bigwedge_{x \in X}\left(1_{x} \rightarrow C(x)\right) \leq B\right\}\right. \\
= & \bigvee\left\{C(x) \mid \bigvee\left(C^{*}(x) \odot F\left(1_{x}^{*}\right)(y) \leq B(y)\right\}\right. \\
= & \bigvee\left\{C(x) \mid C^{*}(x) \leq \bigwedge_{y \in Y}\left(F\left(1_{x}^{*}\right)(y) \rightarrow B(y)\right)\right\} \\
= & \bigvee_{y \in Y}\left(\Delta_{x}(y) \odot B^{*}(y)\right) . \\
e_{L^{Y}}(F(A), B) & =\bigwedge_{y \in Y}\left(F\left(\bigwedge_{x \in X}\left(\left(e_{X}\right)_{x} \rightarrow A(x)\right)\right) \rightarrow B(y)\right) \\
& =\bigwedge_{y \in Y}\left(\bigvee_{x \in X}\left(A^{*}(x) \odot F\left(1_{x}^{*}\right)(y)\right) \rightarrow B(y)\right) \\
& =\bigwedge_{y \in Y} \bigwedge_{x \in X}\left(\left(B^{*}(y) \odot F\left(1_{x}^{*}\right)(y)\right) \rightarrow A(x)\right) \\
& =\bigwedge_{x \in X}\left(\bigvee_{y \in Y}\left(B^{*}(y) \odot \Delta_{x}(y)\right) \rightarrow A(x)\right) \\
& =\bigwedge_{x \in X}(G(B)(x) \rightarrow A(x))=e_{L^{X}}(G(B), A)
\end{aligned}
$$

$(4)(\Rightarrow)$ It follows from Theorem 3.1(4).

$(\Leftarrow)$ Since

$$
F\left(\bigwedge_{x \in X}\left(1_{x} \rightarrow C(x)\right)\right)=F\left(\bigwedge_{x \in X}\left(C^{*}(x) \rightarrow 1_{x}^{*}\right)=\bigwedge\left(C^{*}(x) \rightarrow F\left(1_{x}^{*}\right)(y)\right)\right.
$$


we define

$$
\begin{aligned}
G(B)(x)= & \bigwedge\{C(x) \mid F(C) \geq B\} \\
= & \bigwedge\left\{C(x) \mid F\left(\bigwedge_{x \in X}\left(1_{x} \rightarrow C(x)\right)\right) \geq B\right\} \\
= & \bigvee\left\{C(x) \mid \bigwedge^{*}\left(C^{*}(x) \rightarrow F\left(1_{x}^{*}\right)(y) \geq B(y)\right\}\right. \\
= & \bigvee\left\{C(x) \mid C^{*}(x) \leq \bigwedge_{y \in Y}\left(B(y) \rightarrow F\left(1_{x}^{*}\right)(y)\right)\right\} \\
= & \bigvee_{y \in Y}\left(\Delta_{x}^{*}(y) \odot B(y)\right)^{*} \\
e_{L^{Y}}(B, F(A)) & =\bigwedge_{y \in Y}\left(B(y) \rightarrow F\left(\bigwedge_{x \in X}\left(A^{*}(x) \rightarrow\left(\left(e_{X}\right)_{x}^{-1}\right)^{*}\right)\right)\right) \\
& \left.=\bigwedge_{y \in Y}\left(B(y) \rightarrow \bigwedge_{x \in X}\left(A^{*}(x) \rightarrow F\left(1_{x}\right)^{*}\right)(y)\right)\right) \\
& \left.=\bigwedge_{y \in Y}\left(B(y) \rightarrow \bigwedge_{x \in X}\left(F\left(1_{x}^{*}\right)^{*}(y) \rightarrow A\right) x\right)\right) \\
& =\bigwedge_{y \in Y}\left(\bigwedge_{x \in X}\left(B(y) \odot F\left(1_{x}^{*}\right)^{*}(y) \rightarrow A(x)\right)\right) \\
& =\bigwedge_{y \in Y} \bigwedge_{x \in X}\left(\left(B(y) \odot F\left(1_{x}\right)^{*}\right)^{*}(y) \rightarrow A(x)\right) \\
& =\bigwedge_{x \in X}\left(\bigvee_{y \in Y}\left(B(y) \odot \Delta_{x}^{*}(y)\right)^{*} \rightarrow A(x)\right) \\
& =\bigwedge_{x \in X}(G(B)(x) \rightarrow A(x))=e_{L^{x}}(G(B), A) .
\end{aligned}
$$

EXAMPLE 3.4. Let $X=\{a, b, c\}$ and $Y=\{x, y, z\}$ be sets with

$$
\begin{aligned}
& F_{1}\left(1_{a}\right)(x)=1, F_{1}\left(1_{a}\right)(y)=0.8, F_{1}\left(1_{a}\right)(z)=0.5 \\
& F_{1}\left(1_{b}\right)(x)=0.5, F_{1}\left(1_{b}\right)(y)=0.6, F_{1}\left(1_{b}\right)(z)=0.9, \\
& F_{1}\left(1_{c}\right)(x)=0.3, F_{1}\left(1_{c}\right)(y)=1, F_{1}\left(1_{c}\right)(z)=0.4
\end{aligned}
$$




$$
\begin{aligned}
& F_{2}\left(1_{a}^{*}\right)(x)=1, F_{2}\left(1_{a}^{*}\right)(y)=0.8, F_{2}\left(1_{a}^{*}\right)(z)=0.5, \\
& F_{2}\left(1_{b}^{*}\right)(x)=0.5, F_{2}\left(1_{b}^{*}\right)(y)=0.6, F_{2}\left(1_{b}^{*}\right)(z)=0.9, \\
& F_{2}\left(1_{c}^{*}\right)(x)=0.3, F_{2}\left(1_{c}^{*}\right)(y)=1, F_{2}\left(1_{c}^{*}\right)(z)=0.4 .
\end{aligned}
$$

Define a binary operation $\odot$ (called Łukasiewicz conjection) on $L=$ $[0,1]$ by

$$
x \odot y=\max \{0, x+y-1\}, x \rightarrow y=\min \{1-x+y, 1\} .
$$

(1) If $F_{1}\left(\bigvee_{i \in \Gamma} A_{i}\right)=\bigwedge_{i \in \Gamma} F_{1}\left(A_{i}\right)$ and $F_{1}(\alpha \odot A)=\alpha \rightarrow F_{1}(A)$, then

$$
\begin{aligned}
F_{1}(A)(x)= & \left(A(a) \rightarrow F_{1}\left(1_{a}\right)(x)\right) \wedge\left(A(b) \rightarrow F_{1}\left(1_{b}\right)(x)\right) \\
& \wedge\left(A(c) \rightarrow F_{1}\left(1_{c}\right)(x)\right) \\
= & (A(a) \rightarrow 1) \wedge(A(b) \rightarrow 0.5) \wedge(A(c) \rightarrow 0.3), \\
F_{1}(A)(y)= & (A(a) \rightarrow 0.8) \wedge(A(b) \rightarrow 0.6) \wedge(A(c) \rightarrow 1), \\
F_{1}(A)(z)= & (A(a) \rightarrow 0.5) \wedge(A(b) \rightarrow 0.9) \wedge(A(c) \rightarrow 0.4), \\
G_{1}(B)(a)= & \left(B(x) \rightarrow F_{1}\left(1_{a}\right)(x)\right) \wedge\left(B(y) \rightarrow F_{1}\left(1_{a}\right)(y)\right) \\
& \wedge\left(B(z) \rightarrow F_{1}\left(1_{a}\right)(z)\right) \\
= & (B(x) \rightarrow 1) \wedge(B(y) \rightarrow 0.8) \wedge(B(z) \rightarrow 0.5), \\
G_{1}(B)(b)= & (B(x) \rightarrow 0.5) \wedge(B(y) \rightarrow 0.6) \wedge(B(z) \rightarrow 0.9), \\
G_{1}(B)(c)= & (B(x) \rightarrow 0.3) \wedge(B(y) \rightarrow 1) \wedge(B(z) \rightarrow 0.4) .
\end{aligned}
$$

Thus $\left(e_{L^{X}}, F_{1}, G_{1}, e_{L^{Y}}\right)$ is a Galois connection.

(2) If $F_{1}\left(\bigvee_{i \in \Gamma} A_{i}\right)=\bigvee_{i \in \Gamma} F_{1}\left(A_{i}\right)$ and $F_{1}(\alpha \odot A)=\alpha \odot F_{1}(A)$, then

$$
\begin{aligned}
F_{1}(A)(x)= & \left(A(a) \odot F_{1}\left(1_{a}\right)(x)\right) \vee\left(A(b) \odot F_{1}\left(1_{b}\right)(x)\right) \\
& \vee\left(A(c) \odot F_{1}\left(1_{c}\right)(x)\right) \\
= & (A(a) \odot 1) \vee(A(b) \odot 0.5) \vee(A(c) \odot 0.3), \\
F_{1}(A)(y)= & (A(a) \odot 0.8) \vee(A(b) \odot 0.6) \vee(A(c) \odot 1), \\
F_{1}(A)(z)= & (A(a) \odot 0.5) \vee(A(b) \odot 0.9) \vee(A(c) \odot 0.4),
\end{aligned}
$$




$$
\begin{aligned}
G_{1}(B)(a)= & \left(F_{1}\left(1_{a}\right)(x) \rightarrow B(x)\right) \wedge\left(F_{1}\left(1_{a}\right)(y) \rightarrow B(y)\right) \\
& \wedge\left(F_{1}\left(1_{a}\right)(z) \rightarrow B(z)\right) \\
= & (1 \rightarrow B(x)) \wedge(0.8 \rightarrow B(y)) \wedge(0.5 \rightarrow B(z)), \\
G_{1}(B)(b)= & (0.5 \rightarrow B(x)) \wedge(0.6 \rightarrow B(y)) \wedge(0.9 \rightarrow B(z)), \\
G_{1}(B)(c)= & (0.3 \rightarrow B(x)) \wedge(1 \rightarrow B(y)) \wedge(0.4 \rightarrow B(z)) .
\end{aligned}
$$

Thus $\left(e_{L^{X}}, F_{1}, G_{1}, e_{L^{Y}}\right)$ is a residuated connection.

(3) If $F_{2}\left(\bigwedge_{i \in \Gamma} A_{i}\right)=\bigvee_{i \in \Gamma} F_{2}\left(A_{i}\right)$ and $F_{2}(\alpha \rightarrow A)=\alpha \odot F_{2}(A)$, then

$$
\begin{aligned}
F_{2}(A)(x)= & \left(A^{*}(a) \odot F_{2}\left(1_{a}^{*}\right)(x)\right) \vee\left(A^{*}(b) \odot F_{2}\left(1_{b}^{*}\right)(x)\right) \\
& \vee\left(A^{*}(c) \odot F_{2}\left(1_{c}^{*}\right)(x)\right) \\
= & \left(A^{*}(a) \odot 1\right) \vee\left(A^{*}(b) \odot 0.5\right) \vee\left(A^{*}(c) \odot 0.3\right), \\
F_{2}(A)(y)= & \left(A^{*}(a) \odot 0.8\right) \vee\left(A^{*}(b) \odot 0.6\right) \vee\left(A^{*}(c) \odot 1\right), \\
F_{2}(A)(z)= & \left(A^{*}(a) \odot 0.5\right) \vee\left(A^{*}(b) \odot 0.9\right) \vee\left(A^{*}(c) \odot 0.4\right), \\
G_{2}(B)(a)= & \left(F_{2}\left(1_{a}^{*}\right)(x) \odot B^{*}(x)\right) \vee\left(F_{2}\left(1_{a}^{*}\right)(y) \odot B^{*}(y)\right) \\
& \vee\left(F_{2}\left(1_{a}^{*}\right)(z) \odot B^{*}(z)\right) \\
= & \left(1 \odot B^{*}(x)\right) \vee\left(0.8 \odot B^{*}(y)\right) \vee\left(0.5 \odot B^{*}(z)\right), \\
G_{2}(B)(b)= & \left(0.5 \odot B^{*}(x)\right) \vee\left(0.6 \odot B^{*}(y)\right) \vee\left(0.9 \odot B^{*}(z)\right), \\
G_{2}(B)(c)= & \left(0.3 \odot B^{*}(x)\right) \vee\left(1 \odot B^{*}(y)\right) \vee\left(0.4 \odot B^{*}(z)\right) .
\end{aligned}
$$

Thus $\left(e_{L^{X}}, F_{2}, G_{2}, e_{L^{Y}}\right)$ is a dual Galois connection then

(4) If $F_{2}\left(\bigwedge_{i \in \Gamma} A_{i}\right)=\bigwedge_{i \in \Gamma} F_{2}\left(A_{i}\right)$ and $F_{2}(\alpha \rightarrow A)=\alpha \rightarrow F_{2}(A)$,

$$
\begin{aligned}
F_{2}(A)(x)= & \left(F_{2}\left(1_{a}^{*}\right)^{*}(x) \rightarrow A(a)\right) \wedge\left(F_{2}\left(1_{b}^{*}\right)^{*}(x) \rightarrow A(b)\right) \\
& \wedge\left(F_{2}\left(1_{c}^{*}\right)(x) \rightarrow A(c)\right) \\
= & (0 \rightarrow A(a)) \wedge(0.5 \rightarrow A(b)) \wedge(0.3 \rightarrow A(c)), \\
F_{2}(A)(y)= & (0.2 \rightarrow A(a)) \wedge(0.4 \rightarrow A(b)) \wedge(0.5 \rightarrow A(c)), \\
F_{2}(A)(z)= & (0.5 \rightarrow A(a)) \wedge(0.1 \rightarrow A(b)) \wedge(0.6 \rightarrow A(c)),
\end{aligned}
$$




$$
\begin{aligned}
G_{2}(B)(a)= & \left(F_{2}\left(1_{a}^{*}\right)^{*}(x) \odot B(x)\right) \vee\left(F_{2}\left(1_{a}^{*}\right)^{*}(y) \odot B(y)\right) \\
& \vee\left(F_{2}\left(1_{a}^{*}\right)^{*}(z) \odot B(z)\right) \\
& =(0 \odot B(x)) \vee(0.2 \odot B(y)) \vee(0.5 \odot B(z)), \\
G_{2}(B)(b)= & (0.5 \odot B(x)) \vee(0.4 \odot B(y)) \vee(0.1 \odot B(z)), \\
G_{2}(B)(c)= & (0.7 \odot B(x)) \vee(0 \odot B(y)) \vee(0.6 \odot B(z)) .
\end{aligned}
$$

Thus $\left(e_{L^{X}}, F_{2}, G_{2}, e_{L^{Y}}\right)$ is a dual residuated connection.

\section{References}

[1] R. Bělohlávek, Fuzzy Galois connections, Math. Logic Quart. 45 (1999), 497504.

[2] R. Bělohlávek, Lattices of fixed points of Galois connections, Math. Logic Quart. 47 (2001), 111-116.

[3] A. Frascella, Fuzzy Galois connections under weak conditions, Fuzzy Sets and Systems 172 (2011), 33-50.

[4] G. Georgescu, A. Popescue, Non-dual fuzzy connections, Arch. Math. Logic 43 (2004), 1009-1039.

[5] Ewa. Orlowska, I. Rewitzky, Algebras for Galois-style connections and their discrete duality, Fuzzy Sets and Systems 161 (2010), 1325-1342.

[6] Turunen, Mathematics behind Fuzzy Logic, Physica-Verlag, Heidelberg, (1999).

[7] R. Wille, Restructuring lattice theory; an approach based on hierarchies of concept, in: 1. Rival(Ed.), Ordered Sets, Reidel, Dordrecht, Boston (1982).

[8] W. Yao, L.X. Lu, Fuzzy Galois connections on fuzzy posets, Math. Logic Quart. 55 (2009), 105-112.

Department of Mathematics

Natural Science

Gangneung-Wonju National University

Gangneung, Gangwondo

210-702, Korea

E-mail: yck@gwnu.ac.kr

Department of Applied Mathematics

Pai Chai University

Dae Jeon, 302-735, Korea

E-mail: yskim@pcu.ac.kr 